\title{
Prevalências de doenças crônicas e acesso aos serviços de saúde no Brasil: evidências de três inquéritos domiciliares
}

\author{
Prevalence of chronic diseases and access to health services \\ in Brazil: evidence of three household surveys
}

Taynãna César Simões (https://orcid.org/0000-0002-5849-343X) ${ }^{1}$

Karina Cardoso Meira (http://orcid.org/0000-0002-1722-5703) ${ }^{2}$

Juliano dos Santos (http://orcid.org/0000-0001-9961-3576) ${ }^{3}$

Daniel Cardoso Portela Câmara (https://orcid.org/0000-0002-5439-4477) 4,5

${ }^{1}$ Núcleo de Estudos em Saúde Pública e Envelhecimento (NESPE), Instituto René Rachou, Fundação Oswaldo Cruz. Av. Augusto de Lima 1.715, Barro Preto. 30190-002 Belo Horizonte MG Brasil. taynana.simoes@fiocruz.br ${ }^{2}$ Escola de Saúde, Universidade Federal do Rio Grande do Norte. Natal RN

Brasil.

${ }^{3}$ Instituto Nacional do

Câncer, Hospital do Câncer III. Rio de Janeiro RJ Brasil.

${ }^{4}$ Laboratório de Mosquitos Transmissores de

Hematozoários, Instituto Oswaldo Cruz, Fundação

Oswaldo Cruz. Rio de Janeiro RJ Brasil.

${ }^{5}$ Núcleo Operacional

Sentinela de Mosquitos

Vetores, Fundação Oswaldo

Cruz. Rio de Janeiro RJ

Brasil.

\begin{abstract}
Chronic non-communicable diseases (NCDs) are the leading causes of death globally, impacting heavily on the most vulnerable populations. This study aimed to analyze changes in the prevalence of these diseases, health conditions, access, and health services in Brazil between 2008 and 2019. Tests of differences and generalized linear models were used as analytical tools, considering complex sampling from the PNAD 2008, PNS 2013, and PNS 2019 surveys, to test temporal changes in the prevalence and the prevalence ratio estimates, adjusted by sociodemographic variables. An increase in the prevalence of Depression, Diabetes, Cancers, Neuropsychiatric Disorders, Chronic Pulmonary problems, and Musculoskeletal problems was observed. A decline in rheumatoid arthritis, chronic renal failure, and diseases of the circulatory system was identified. Among Brazilians with at least one NCD, an increase in coverage by the family health strategy over time was observed. However, there was a reduction in timely medical care and obtaining of free prescription drugs.

Key words Chronic noncommunicable diseases, Surveys, Generalized linear models, Access to health
\end{abstract}

Resumo As Doenças Crônicas Não Transmissíveis (DCNT) são as principais causas de morte no mundo, impactando fortemente sobre populações mais vulneráveis. O objetivo deste estudo foi analisar as mudanças nas prevalências dessas doenças, nas condições de saúde, acesso e utilização de serviços de saúde no Brasil, entre 2008 e 2019. Como ferramenta analítica, foram utilizados testes de diferenças de proporções e modelos lineares generalizados, considerando amostragem complexa dos inquéritos da Pesquisa Nacional por Amostra de Domicílios (PNAD) de 2008, e da Pesquisa Nacional de Saúde (PNS), nos anos de 2013 e 2019, para testar mudanças no tempo das prevalências e estimar razões de prevalência, ajustadas por variáveis sociodemográficas. Houve aumento das prevalências de depressão, câncer, diabetes, distúrbios neuropsiquiátricos, problemas pulmonares crônicos e problemas osteomusculares. Houve redução de artrite reumatoide, insuficiência renal crônica e doenças do aparelho circulatório. Entre os brasileiros com pelo menos uma DCNT verificou-se aumento da cobertura da Estratégia de Saúde da Família ao longo do tempo, no entanto verificou-se redução do atendimento médico em tempo oportuno e obtenção de medicamentos prescritos gratuitamente.

Palavras-chave Doenças crônicas não Transmissíveis, Inquéritos, Acesso à saúde, modelos lineares generalizados 


\section{Introdução}

As Doenças Crônicas Não Transmissíveis (DCNT) representam as principais causas de adoecimento e morte no mundo. O aumento da carga de doenças por esse grupo de causas está relacionado ao envelhecimento populacional, mudanças nos hábitos e estilo de vida, e disparidades socioeconômicas e de acesso aos serviços de saúde. Essas doenças englobam várias condições de saúde que apresentam em comum sua origem multifatorial, associado à exposição prolongada a fatores de risco modificáveis, que promovem lesões, incapacidades e óbitos ${ }^{1-5}$.

A Organização Mundial da Saúde (OMS) considera como DCNT somente as doenças do aparelho circulatório (DAC), neoplasias ou cânceres (CA), doenças respiratórias crônicas (DRC) e diabetes mellitus (DM), por apresentarem em sua história natural fatores de risco e de proteção em comum, facilitando o desenvolvimento de políticas de prevenção e controle ${ }^{6,7}$. No entanto, outras condições crônicas de saúde definem-se como DCNT pela definição da OMS, tendo também impacto importante na carga de doenças e nas estimativas de anos de vida perdidos por incapacidade, tais como as doenças osteomusculares relacionadas ao trabalho (DORT) e articulares, insuficiência renal crônica (IRC), e distúrbios neuropsiquiátricos $(\mathrm{DN})^{1,7}$. Em 2013, 66 milhões de brasileiros $(45,1 \%)$ referiram ao menos uma DCNT, excluindo-se a hipertensão arterial sistêmica (HAS), sendo os mais prevalentes problemas na coluna $(18,5 \%)$, depressão $(7,6 \%)$, artrite $(6,4)$ e DM $(6,2 \%)$. Aquelas que promoveram grau de limitação muito intenso ou intenso foram os $\mathrm{DN}(49,4 \%)$, problemas na coluna e DORT $(32,1 \%)^{8}$.

Os países desenvolvidos apresentam as maiores taxas de incidência de DCNT, no entanto, devido às desigualdades de acesso à alimentação, educação e atenção à saúde, $80 \%$ dos óbitos ocorrem em países de baixa e média renda, com mais de 30\% das mortes, ocorrendo em indivíduos com menos de 60 anos de idade ${ }^{1,2}$. Oitenta por cento das mortes por DCNT no mundo são devido às DAC, CA, DRC e DM $\mathrm{DM}^{1,2,5}$.

A história natural dessas doenças e seu tratamento podem gerar incapacidades, reduzindo a renda do indivíduo e da família, acentuando as desigualdades socioeconômicas e de saúde. Além disso, impactam no Sistema de Saúde, devido às necessidades de cuidados mais prolongados e dispendiosos ${ }^{6,7}$. No Brasil, estima-se que $72 \%$ dos óbitos sejam por DCNT, estando associados aos principais fatores de risco modificáveis, além das desigualdades socioeconômicas, dificuldade de acesso aos serviços de saúde, deficiências em educação e informação em saúde ${ }^{8-10}$.

$\mathrm{Na}$ última década, houve aumento da mortalidade por CA e DM, e redução das taxas de mortalidade por DAC e DRC no Brasil ${ }^{8,9}$, potencialmente relacionados à redução da prevalência do tabagismo na população, ampliação do acesso à saúde após a implantação do Sistema Único de Saúde (SUS), expansão da Atenção Básica (AB), e acesso ampliado a medicamentos essenciais. Esse perfil de redução das taxas de mortalidade não é uniforme no território brasileiro, sendo maior nas regiões que apresentam maior desenvolvimento socioeconômico e acesso aos serviços de saúde ${ }^{8,9,11,12}$.

Com o objetivo de reduzir tanto a mortalidade por DCNT, quanto as prevalências dos principais fatores de risco modificáveis (tabagismo, álcool em excesso, inatividade física e hábitos alimentares não saudáveis), o Ministério da Saúde (MS) apresentou o Plano de Ações Estratégicas para o Enfrentamento das Doenças Crônicas Não Transmissíveis (DCNT) no Brasil, 2011-2022, com o intuito de promover o desenvolvimento e a implementação de políticas públicas efetivas, integradas e sustentáveis, baseadas em evidências, para a prevenção e controle das DCNT, entre as quais: acidente vascular cerebral (AVC), infarto agudo do miocárdio (IAM), hipertensão arterial (HA), CA, DM, e DRC. O Plano apresenta como pilares a vigilância, informação, avaliação e monitoramento, promoção à saúde, e cuidado integral $^{14}$. Entre as ações já realizadas destacam-se a Academia da Saúde, Farmácia Popular, expansão da Atenção Básica e Estratégia de Saúde da Família (ESF), Rede de Urgência e Emergências, e Redes de Atenção das DCNT ${ }^{13}$.

Os Inquéritos Nacionais de Saúde (INS) permitem conhecer o perfil de saúde e a distribuição dos fatores de risco em uma população, com atualização periódica e comparações sequenciadas no tempo e entre áreas geográficas ${ }^{14}$. Frente à necessidade de vigilância contínua das DCNT, o presente estudo visa avaliar as mudanças temporais das prevalências de doenças crônicas e do acesso e utilização de serviços de saúde, entre inquéritos domiciliares de âmbito nacional realizados em 2008, 2013 e 2019, no Brasil. O estudo avalia as DCNT definidas pela OMS, além dos distúrbios neuropsiquiátricos (DN), desordens osteomusculares (DORT) e articulares, e insuficiência renal crônica (IRC). 


\section{Métodos}

\section{Desenho de estudo e fontes de dados}

Trata-se de um estudo transversal em painéis para avaliação de mudanças temporais nas prevalências de doenças crônicas, condições de saúde e de acesso e utilização de serviços de saúde no Brasil, segundo informações autorreferidas de inquéritos domiciliares. Foram avaliadas as mudanças no tempo e influências de fatores sociodemográficos sobre as prevalências, além de descritos motivos de não acesso a atendimento e medicamentos prescritos. Os dados foram obtidos da Pesquisa Nacional por Amostra de Domicílios (PNAD) de 2008, e da Pesquisa Nacional de Saúde (PNS), nos anos de 2013 e 2019, pelo Instituto Brasileiro de Geografia e Estatística $(\text { IBGE })^{15-17}$.

A PNAD tem periodicidade anual e faz parte do sistema de pesquisas domiciliares do Brasil, tendo informações básicas para o estudo do desenvolvimento socioeconômico do país. Regularmente são feitas pesquisas adicionais sobre alguns temas, como a Pesquisa Suplementar de Saúde. Com intervalos de cinco anos, o intuito é produzir dados de base populacional sobre acesso e cobertura de planos de saúde, e estimar as prevalências de alguns agravos à saúde e outros fatores autorreferidos. A amostra é probabilística, obtida em três estágios (municípios, setores censitários, domicílios), e representativa para Brasil, Grandes Regiões, e Unidades da Federação ${ }^{15}$.

A PNS com periodicidade de cinco anos apresenta como eixos principais o desempenho do sistema nacional de saúde, as condições de saúde da população brasileira e a vigilância das doenças crônicas não transmissíveis e fatores de risco associados ${ }^{14}$. As amostras probabilísticas são constituídas por estratificação e conglomeração em três estágios (setores censitários, domicílios, indivíduo maior de 18 anos) $)^{16,17}$.

\section{Base de dados integrada}

Foram utilizadas variáveis de identificação dos indivíduos e domicílios, variáveis dos planos amostrais, variáveis sociodemográficas, de doenças crônicas (exceto HAS), de cobertura por plano de saúde, de acesso e utilização dos serviços de saúde, estratificados por tipo de serviço (público ou privado) e tipo de obtenção (pagamento/plano de saúde/SUS), dos módulos Q e J dos questionários da PNS 2019 e PNS 2013, e do Suplemento Saúde da PNAD 2008. Foram sele- cionadas variáveis comuns nos três inquéritos, ou entre as PNS, criando uma base de dados integrada, após harmonização das variáveis (limpeza, completude, codificação de nomes, padronização de categorias de resposta).

A população analisada correspondeu a todos os indivíduos participantes dos inquéritos, com idade acima de 18 anos, que foram selecionados (sorteados nas PNS ou morador do domicílio respondente sendo a própria pessoa ou outro morador do domicílio no bloco de características de saúde na PNAD), e que tiveram o status de entrevista realizada.

Nos três inquéritos foram considerados os indivíduos que referiram ter tido diagnóstico médico para as seguintes DCNT, classificadas em cinco grupos de doenças e condições crônicas: (1) Doenças do Aparelho Circulatório (DAC): DAC geral (Infarto, Angina, Insuficiência cardíaca, entre outras); Infarto; Angina; Insuficiência cardíaca; Acidente Vascular Cerebral (AVC); (2) Doenças Neuropsiquiátricas (DN): Depressão; Esquizofrenia; Transtorno Bipolar; Transtorno Obsessivo Compulsivo (TOC); Ter pelo menos uma das três últimas (Outras DN); (3) Neoplasias (CA): Qualquer câncer; Câncer de Pele; Pulmão; Intestino; Estômago; Mama, Colo do Útero; Próstata; (4) Outras DCNT: Alguma doença pulmonar crônica (enfisema pulmonar, bronquite crônica, DPOC - Doença pulmonar obstrutiva crônica); Asma ou Bronquite; Diabetes Mellitus (DM); Artrite e reumatismo; Problema Crônico de Coluna/Costas (Algum problema crônico de coluna, como dor crônica nas costas ou no pescoço, lombalgia, dor ciática, problemas nas vértebras ou disco); DORT; Insuficiência Renal Crônica (IRC).

Dentre os indivíduos que relataram ter pelo menos uma das DCNT comuns nos três inquéritos (DAC geral, Depressão, Algum Câncer, Asma ou Bronquite, Diabetes, Artrite e Reumatismo ou IRC), foram observadas algumas condições de saúde como: autopercepção do estado de saúde (Muito Boa/Boa, Regular/Ruim/Muito Ruim); interrupção de atividades habituais por motivos de saúde nas duas últimas semanas (Não/Sim); se esteve acamado nas últimas duas semanas (Não/ Sim).

O acesso a serviços de saúde pode ser medido pela procura e utilização dos mesmos, incluindo assistência, consultas de rotina ou de emergência, obtenção de medicamentos prescritos ${ }^{18}$. Dentre os portadores de pelo menos uma das DCNT comuns nos três inquéritos, o acesso e utilização de serviços de saúde foram medidos pelas pre- 
valências de pessoas com plano de saúde; cujo domicílio é atendido pelo Programa Estratégia Saúde da Família (ESF); com hábito de procurar sempre o mesmo médico ou o serviço de saúde; que se consultou com médico nos últimos 12 meses; que procurou e que tenha sido atendido em até duas tentativas nas duas semanas anteriores à pesquisa por motivo de doença; de prescrição e obtenção de medicamentos; de internação nos últimos 12 meses, que tenha utilizado serviço de emergência domiciliar e uso de ambulância.

Algumas dessas variáveis foram estratificadas por tipo do serviço (público/particular) ou forma de obtenção da assistência/medicamento conforme informações de cobertura por plano de saúde, pagamento de algum valor, e se foi feito por serviço público ou SUS (pago/plano/SUS). Foram descritos alguns motivos de não acesso à assistência de saúde e não obtenção dos medicamentos prescritos.

As variáveis sociodemográficas utilizadas foram: Macrorregião de residência - Nordeste $(\mathrm{NE})$, Centro-Oeste $(\mathrm{CO})$, Norte $(\mathrm{N})$, Sudeste (SE), Sul (S); Idade (18 a 39, 40 a 59, 60 anos ou mais); Sexo (Feminino/Masculino); Escolaridade (Até fundamental completo, Ensino médio ou superior); Raça/cor (Branca, Não Branca); Zona de Residência (Rural/Urbana).

\section{Análise estatística}

O primeiro passo da análise estatística foi atribuir o desenho amostral, utilizando os diferentes pesos e efeitos de desenho dos três inquéritos, agora em uma base de dados única, considerando um termo de interação entre o estrato amostral e o período de observação na definição do desenho amostral ${ }^{19}$.

As prevalências das DCNT, de condições de saúde, e de variáveis de acesso e utilização de saúde foram estimadas com os respectivos intervalos de $95 \%$ de confiança, nos períodos dos inquéritos e segundo variáveis sociodemográficas. As diferenças entre essas prevalências foram testadas por meio do teste qui-quadrado com correção de Rao Scott, que considera pesos amostrais e efeito de desenho nos cálculos ${ }^{20}$. A significância das diferenças foram avaliadas através dos p-valores destes testes, e não pela sobreposição de intervalos de confiança entre as categorias das variáveis, prática que pode levar a evidências errôneas, uma vez que há aumento da probabilidade de detectar diferenças não verdadeiras (Erro do Tipo I). Algumas variáveis constavam apenas nas PNS, assim as diferenças temporais foram avaliadas apenas entre 2013 e 2019. Foram estimadas prevalências referentes aos motivos de não acesso ou uso de serviço de saúde ou de não obtenção de medicamentos prescritos para pelo menos uma das condições crônicas.

Posteriormente, foram estimadas as Razões de Prevalência e Intervalos de 95\% de Confiança das DCNT, das condições de saúde, e de variáveis de acesso e utilização de serviços de saúde, através de Modelos Lineares Generalizados (GLM) com distribuição de probabilidade Poisson ${ }^{21}$. A variável independente principal do estudo foi o ano de realização do inquérito, e as variáveis sociodemográficas macrorregião de residência, idade e sexo foram utilizadas para ajuste. Entre as DCNT, foram também avaliadas as significâncias de termos de interação das variáveis sociodemográficas com $\mathrm{o}$ ano, a fim de avaliar mudanças nas prevalências em subgrupos populacionais ao longo do tempo. Todas as estimativas pontuais, intervalares, testes de diferenças de proporções, e modelos de regressão consideraram os pesos amostrais e correção para efeito de desenho ${ }^{22}$, através do pacote survey ${ }^{23}$ do software estatístico $\mathrm{R}^{24}$.

\section{Resultados}

A base integrada foi formada pelo somatório de 391.868, 205.546, 293.725 indivíduos das PNAD 2008, PNS 2013 e PNS 2019, respectivamente. Desses, 97.589 tiveram pelo menos uma dentre DAC geral, Depressão, algum Câncer, Asma ou Bronquite, Diabetes, Artrite e Reumatismo ou IRC. Os resultados deste estudo serão descritos por blocos de grupos de DCNT, condições de saúde, e acesso e utilização de serviços de saúde, destacando apenas diferenças estatisticamente significativas.

A Tabela 1 apresenta as estimativas pontuais e intervalares das prevalências e Razões de Prevalência (RP) das DCNT de pelo menos dois inquéritos, bem como os testes de diferenças entre os anos. As RP foram ajustadas segundo região de residência, idade e sexo, sendo a base de comparação o ano de 2008 para doenças avaliadas nos três anos, e 2013 para doenças observadas apenas nas PNS. A Figura 1 mostra as estimativas pontuais e intervalares das prevalências em cada grupo de doenças crônicas, segundo as variáveis sociodemográficas. As interações significativas entre estas variáveis e ano foram descritas. Na Tabela 2 são apresentadas as prevalências segundo ano e as Razões de Prevalência (RP) ajustadas para as condições de saúde, e variáveis de acesso e uti- 


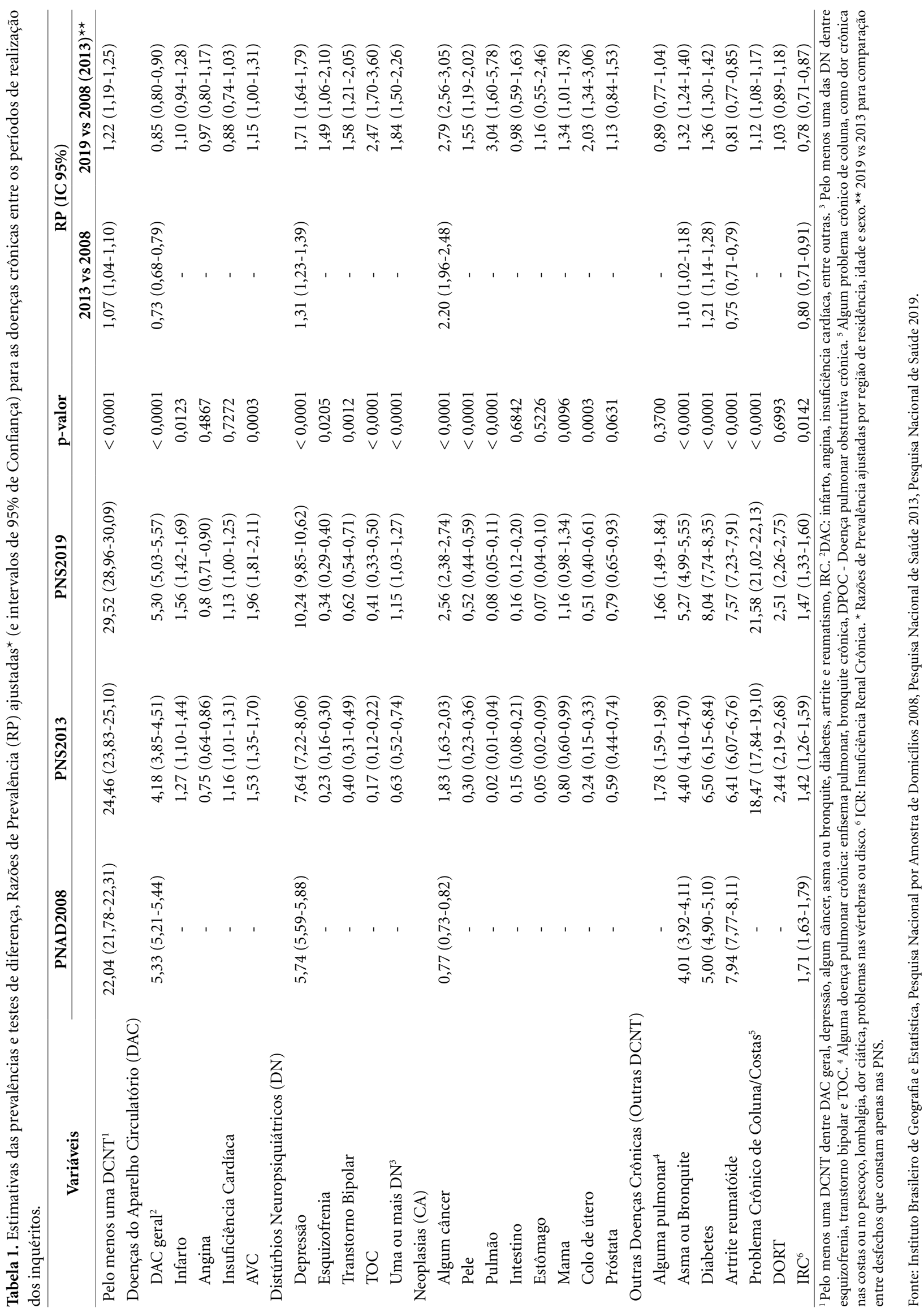


Doenças do Aparelho Circulatório
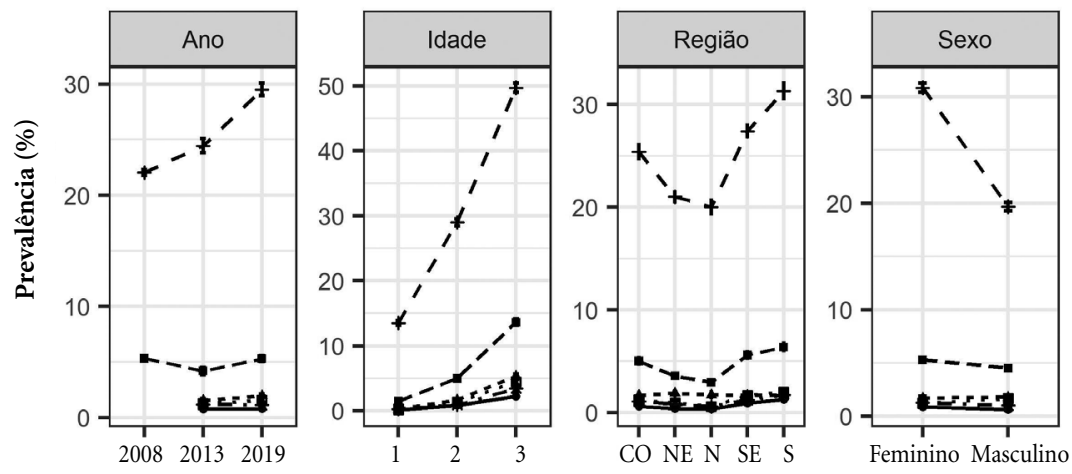

Variáveis socioeconômicas

D.A.C.

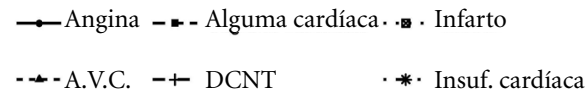

Distúrbios Neuropsiquiátricos
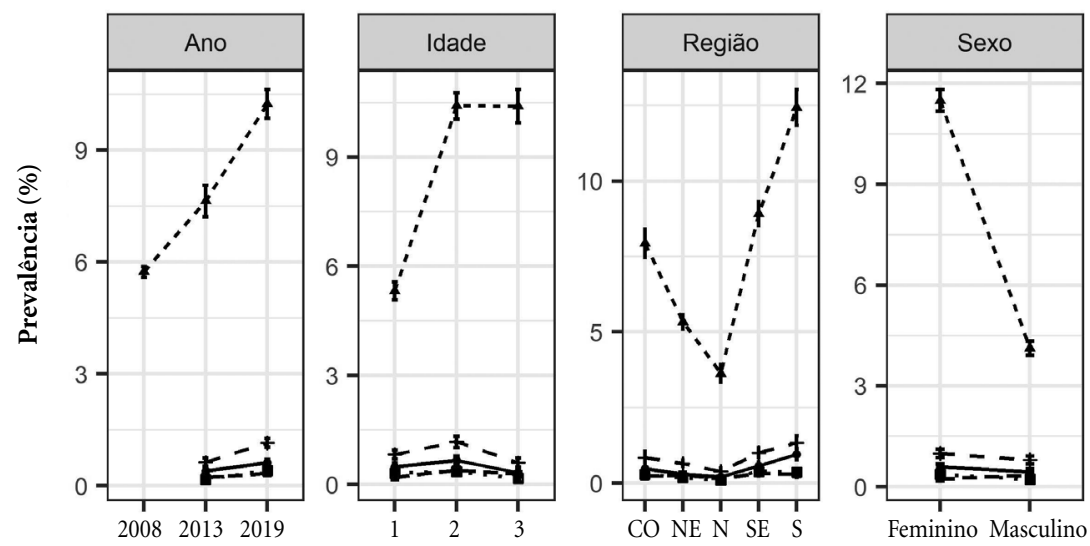

Variáveis socioeconômicas

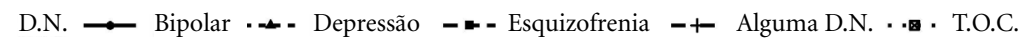

continua

Figura 1. Prevalência de cada grupo de doenças crônicas, segundo as variáveis sociodemográficas, região de residência, idade, sexo, escolaridade e ano do inquérito / Legenda: Idade :1 (18-39 anos), 2 (40-59 anos) e 3 ( $\geq 60$ anos).

lização de serviços de saúde entre os indivíduos que apresentaram pelo menos uma das DCNT observadas nos três inquéritos. A Figura 2 apresenta as distribuições de frequência de motivos de não acesso à saúde considerando atendimento e não obtenção de medicamentos prescritos.

A prevalência de ter pelo menos uma das DCNT observadas nos três inquéritos (DAC geral, depressão, algum câncer, asma ou bronquite, diabetes, artrite e reumatismo, IRC) aumentou significativamente entre os períodos, variando de 22,40 a 29,52\% entre 2008 e 2019 (Tabela 1).

\section{Doenças do aparelho circulatório (DAC)}

Houve diminuição na prevalência de DAC geral de 2008 a 2013, voltando a crescer em 2019, enquanto infarto e AVC cresceram de 2013 a 2019 (Tabela 1). DAC é mais prevalente no S, SE e CO, infarto e angina no SE e $\mathrm{S}$, e insuficiência cardíaca no $\mathrm{S}$ (Figura 1). As DAC diminuíram em 2013 no N e aumentaram no SE. Em 2019, houve diminuição no CO de DAC, infarto e insuficiência cardíaca, que também diminuiu no $S$. As DAC aumentam com a idade, apresentando 

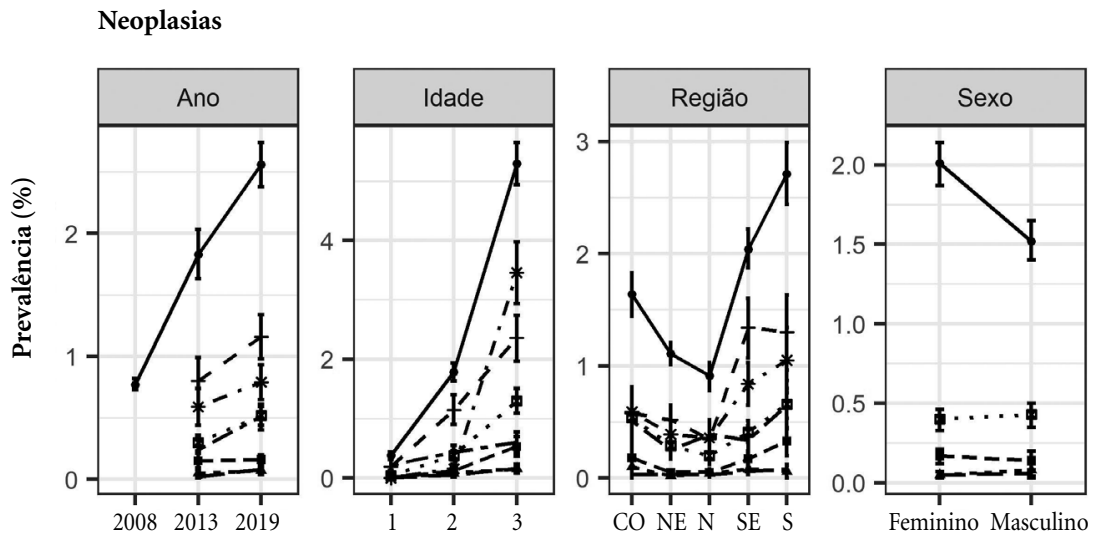

Variáveis socioeconômicas

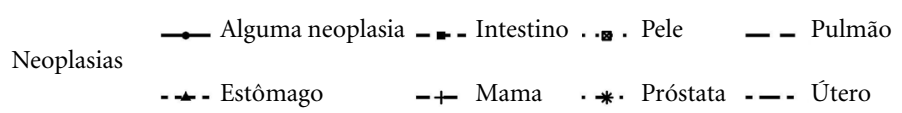

Outras doenças crônicas não transmissíveis
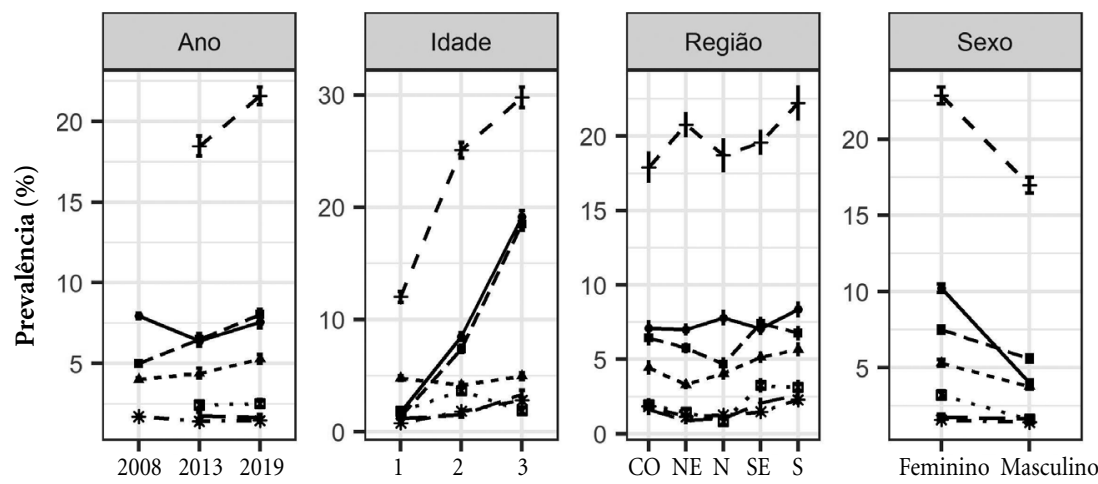

Variáveis socioeconômicas

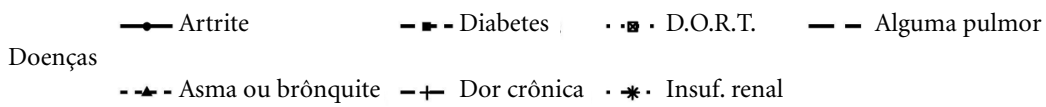

Figura 1. Prevalência de cada grupo de doenças crônicas, segundo as variáveis sociodemográficas, região de residência, idade, sexo, escolaridade e ano do inquérito / Legenda: Idade :1 (18-39 anos), 2 (40-59 anos) e 3 ( $\geq 60$ anos).

Fonte: Instituto Brasileiro de Geografia e Estatística, Pesquisa Nacional por Amostra de Domicílios 2008, Pesquisa Nacional de Saúde 2013, Pesquisa Nacional de Saúde 2019.

altos valores acima de 60 anos, em pessoas da raça/cor branca (sem diferença para DAC geral e AVC), com baixo grau de instrução, e residentes em urbana (sem diferença para angina e AVC). Em 2013, DAC geral diminuiu em maiores de 60 anos e aumentou na área urbana, enquanto infarto diminui na faixa etária de 40 a 59 anos. Em 2019, DAC geral diminuiu em maiores de 40 anos e nos indivíduos menos escolarizados. Angina diminuiu em menos escolarizados, e AVC aumentou na área rural. Mulheres têm maior prevalência de DAC geral, angina, insuficiência cardíaca, enquanto homens têm maior prevalência de infarto. Em 2019, houve aumento de DAC geral entre homens. 


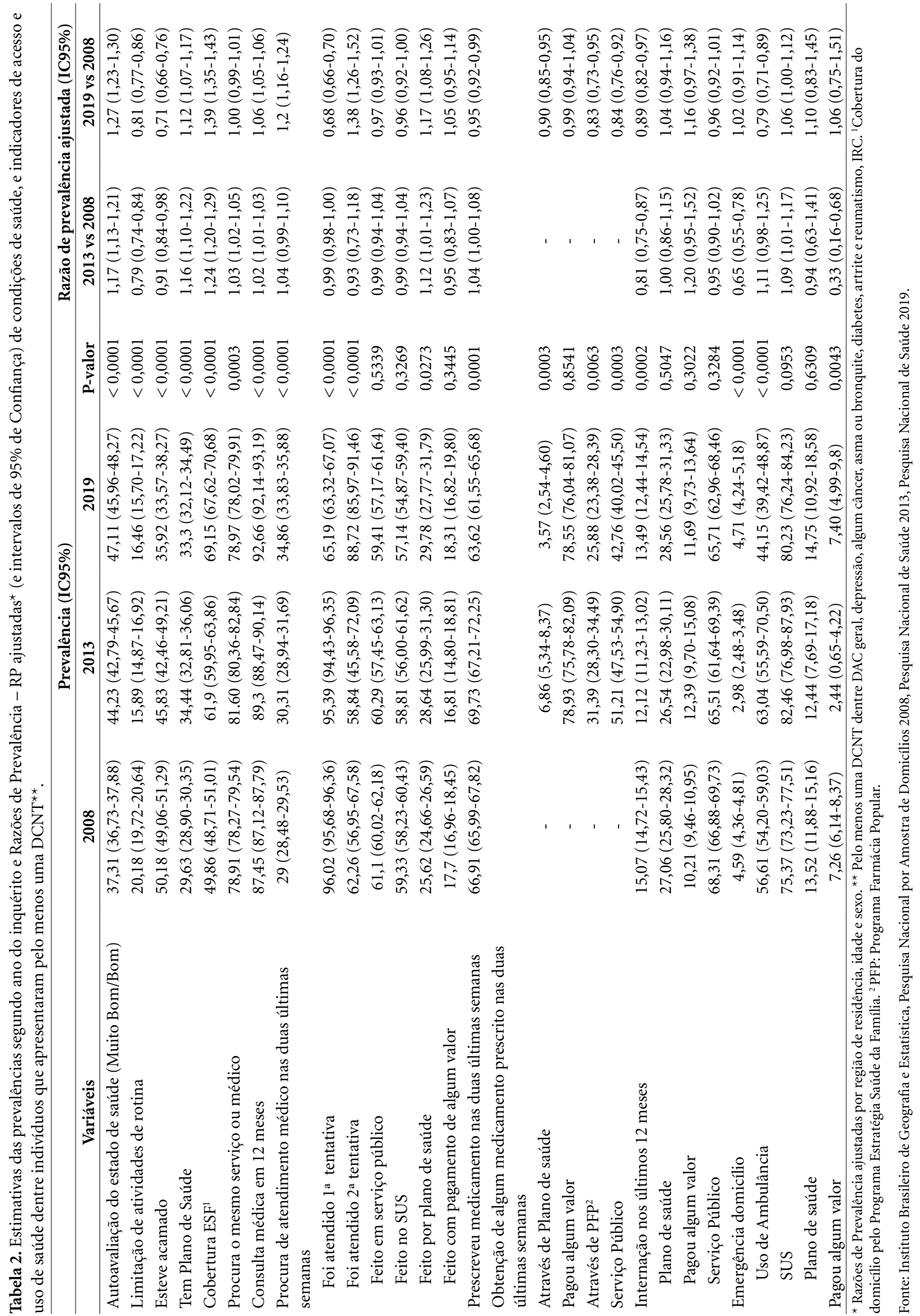




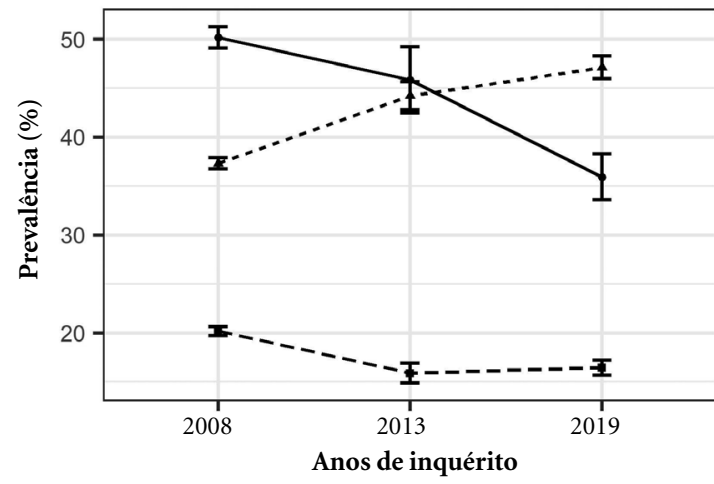

Condições de saúde

$\rightarrow$ Acamado

- ... Estado de saúde

-2- Limit. ativ.

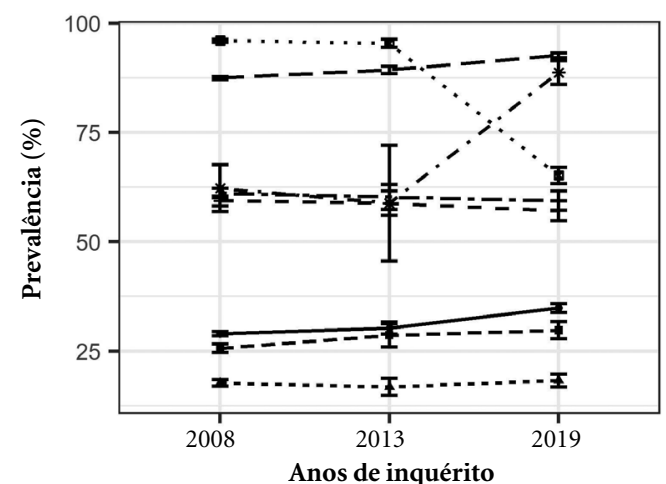

Atendimento e local

Atend. 2 sem.

- - - Atend. pagou

-- Atend. plano

-+ Atend. SUS

. Atend. la vez

* Atend. 2a vez

- - Consulta med. 12 meses

- - - Atend. serv. público

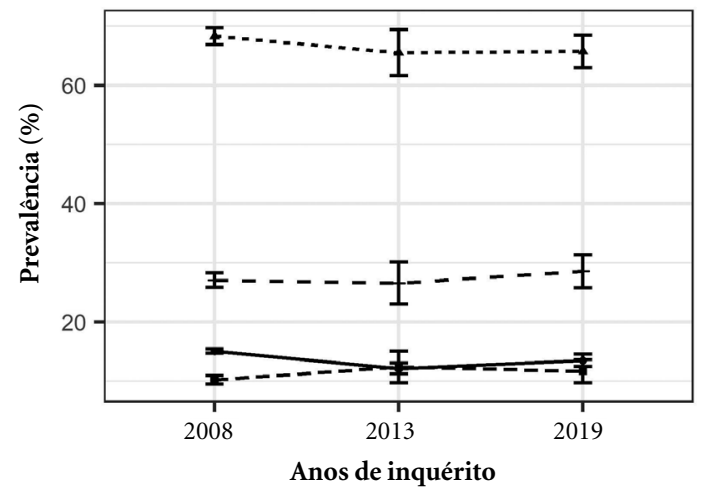

Internação

$\rightarrow$ Inter. 12 meses

- ـ - Intern. serv. público

-.- Intern. pagou

-+ Intern. plano

continua

Figura 2. Prevalência de acesso aos serviços de saúde, medicamentos e condição de saúde, segundo ano do inquérito.

\section{Distúrbios neuropsiquiátricos (DN)}

Houve aumento importante da prevalência de depressão, e para os demais DN (esquizofrenia, transtorno bipolar e TOC) no período. A prevalência de ter uma dentre esses três DN passou de $0,63 \%$ em 2013 para 1,15\% em 2019 (Tabela 1). A depressão teve maior prevalência no S, seguido do SE e CO (Figura 1). O Transtorno Bipolar tem maior prevalência no $\mathrm{S}$. Em 2019, houve diminuição deste DN no SE. De- pressão aumenta com a idade, sendo mais alta a partir dos 40 anos. O mesmo é observado para esquizofrenia, embora haja uma tendência de queda a partir dos 60 anos, faixa etária em que o transtorno bipolar é menos frequente. Em 2013, houve diminuição da depressão entre maiores de 40 anos e em indivíduos menos escolarizados, e aumento de esquizofrenia entre maiores de 60 anos. Em 2019, a diminuição da depressão se manteve, além da diminuição entre homens. Em 2019 houve aumento de esquizofrenia na faixa de 


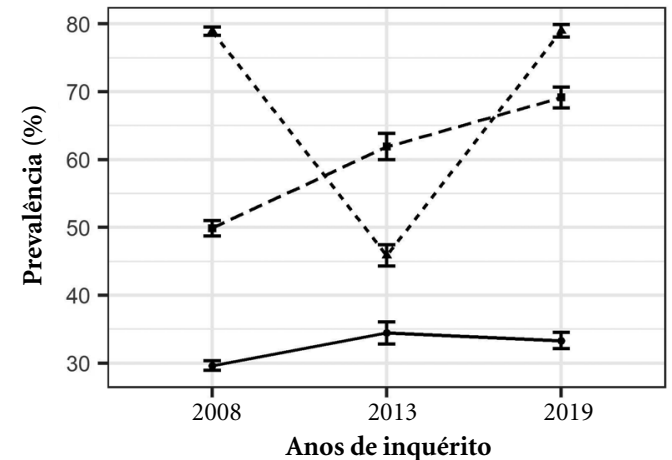

Cobertura

$\rightarrow$ Plano de saúde

. _. Procura mesmo serv.

- - - Cob. ESF

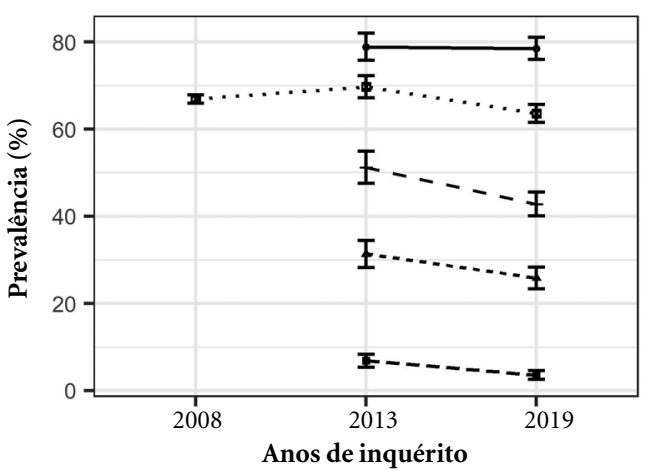

Medicamentos

$\rightarrow$ Medicam. pagou

_. - Medicam. PFP

-2- Medicam. plano

-+ Medicam. serv. público

.. Medicam. receitado

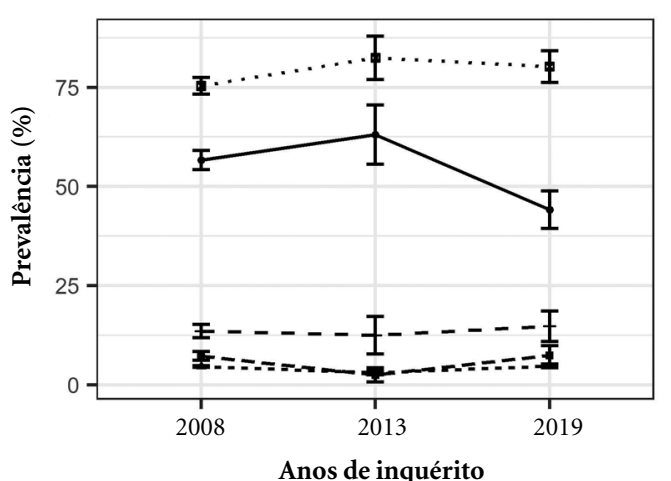

Atendimento domiciliar

$\rightarrow$ Domicílio ambulância

_._. Domicílio 12 meses

-.- Domicílio pagou

-+ Domicílio plano

* Domicílio SUS

Figura 2. Prevalência de acesso aos serviços de saúde, medicamentos e condição de saúde, segundo ano do inquérito.

Fonte: Instituto Brasileiro de Geografia e Estatística, Pesquisa Nacional por Amostra de Domicílios 2008, Pesquisa Nacional de Saúde 2013, Pesquisa Nacional de Saúde 2019.

40 e 59 anos. Mulheres têm maior prevalência de depressão, transtorno bipolar e TOC, e homens de esquizofrenia. Depressão e esquizofrenia são mais prevalentes entre indivíduos com menor escolaridade, enquanto transtorno bipolar e TOC ocorrem mais entre os mais escolarizados. Os DN são mais frequentes para raça/cor branca (sem diferença para esquizofrenia), e em residentes na zona urbana.

\section{Neoplasias (CA)}

Todos os tipos de cânceres aumentaram com o ano e a idade, com exceção de câncer de intestino e estômago (Tabela 1 e Figura 1). Houve alta prevalência entre maiores de 60 anos para câncer de pele, pulmão, próstata, e de estômago acima dos 40 anos (Figura 1). S e SE tiveram alta prevalência de câncer de pele, e o CO e S de câncer de colo do útero. Em 2019, houve diminuição de câncer de colo de útero no N. A maioria dos 


\section{Outras doenças crônicas}

Dentre as demais DCNT, houve aumento no período na prevalência de asma ou bronquite, diabetes, artrite e reumatismo (entre 2013 e 2019), e problema crônico de costas e coluna (Tabela 1). Houve redução de IRC e artrite reumatoide. As prevalências da maioria dessas doenças aumentam com o avançar da idade, especialmente para maiores de 60 anos, em indivíduos da cor branca, com baixa escolaridade (mais escolarizados têm mais asma), e residentes da área urbana (rural tem mais DORT e problema crônico de coluna/costas) (Figura 1). Observou-se alta prevalência de doenças respiratórias nas regiões $\mathrm{S}, \mathrm{SE}$ e CO, mais IRC no CO e S, mais DM e DORT no S e SE. Em 2013, houve aumento de asma no $\mathrm{Ne}$ diminuição no $S$ até 2019. Em 2019 houve queda de asma e diabetes no SE, e desta última no $\mathrm{N}$ e S. Mulheres tiveram maior prevalência de asma, diabetes, artrite e reumatismo, problema crônico na coluna/costas e DORT.

\section{Condições de saúde e acesso e utilização de serviços de saúde}

Houve aumento das prevalências de indivíduos com autoavaliação do estado de saúde Boa/ Muito boa, e diminuição das prevalências de indivíduos que relataram limitações para realizar atividades de rotina devido à doença e de indivíduos que estiveram acamados nas duas últimas semanas. A proporção de indivíduos com plano de saúde teve um aumento importante de 2008 a 2013, não tendo aumento aparente no próximo período. Domicílios cobertos pelo programa Estratégia Saúde da Família apresentou aumento importante entre todos os períodos.

Dentre as variáveis de acesso e utilização dos serviços de saúde, $78 \%$ tinham o hábito de procurar o mesmo serviço ou médico em 2019. Houve aumento do número de consultas médicas realizadas em 12 meses. Houve aumento da procura de atendimento médico em duas semanas, mas diminuição ao longo do tempo de atendimen- tos realizados na primeira tentativa de busca. A prevalência de atendimentos feitos na segunda tentativa aumentou de $62,26 \%$ para $88,72 \%$ no período. Não houve diferença da prevalência de atendimentos feitos em serviço público, pelo SUS ou que tenha sido pago ao longo do tempo, em contrapartida ao aumento de atendimento feitos por plano de saúde. Houve diminuição da prevalência de medicamentos prescritos. Dentre os medicamentos prescritos, houve diminuição da obtenção por meio de plano de saúde, Programa Farmácia Popular e Serviço Público, se mantendo constante a alta proporção de obtenção de medicamentos por pagamento. Diminuiu a prevalência de internações em 12 meses, e aumentou as emergências domiciliares via SUS, porém com diminuição de transporte por ambulâncias.

Dentre os indivíduos com pelo menos uma DCNT, a Figura 3 mostra os motivos para não procurar atendimento ou de não obtenção dos medicamentos prescritos. Os principais motivos para não ter sido atendido na primeira procura por atendimento médico nas últimas duas semanas, foram falta de dinheiro, seguido de ausência de médico ou profissional, e falta de vaga para atendimento. No entanto, observa-se que tanto a desistência pelo atendimento quanto a inoperância da unidade ou do equipamento em questão mantiveram-se constantes ao longo dos três inquéritos. Ao analisar o motivo pelo qual não foi atendido na última vez que procurou atendimento nas últimas duas semanas (segunda tentativa de atendimento), quase todas as categorias apresentam acréscimo ao longo do tempo. A falta de dinheiro só foi representada em 2008. Dentre os motivos de não obtenção dos medicamentos prescritos, há padrões distintos: não achar necessário e falta de acesso (seja pela falta de proximidade ou por não disponibilidade do medicamento na farmácia) aumentam ao longo do tempo, enquanto não ter dinheiro, sentir-se melhor e outros motivos caíram em 2013, mas com tendência de aumento em 2019. Dentre os motivos para não ter procurado serviço de saúde nas duas últimas semanas, há queda de 2008 para 2013, com posterior aumento ou estabilidade entre 2013 e 2019 para praticamente todas as categorias de respostas (Figura 3).

\section{Discussão}

Com o envelhecimento da população brasileira, as DCNT passaram a representar alta carga de doenças no país, com demanda significativa para 
Motivo não foi atendido na primeira vez que procurou atendimento nas duas últimas semanas

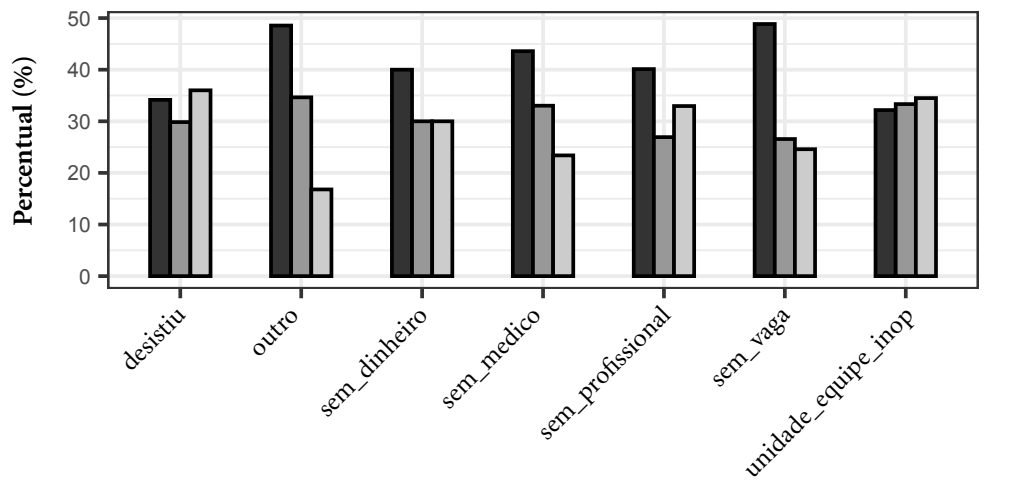

Categorias de respostas Anos de inquérito $\square 2008$ 2013 2019

Motivo não foi atendido na última vez que procurou atendimento nas duas últimas semanas

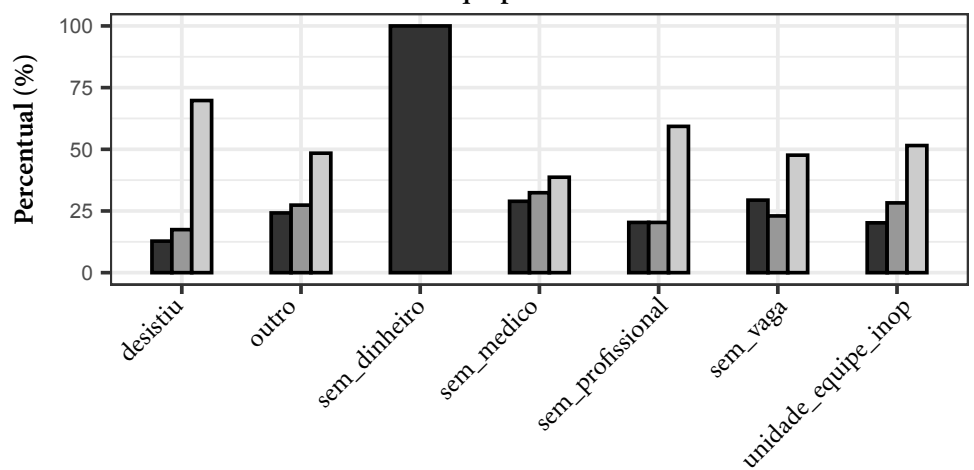

Categorias de respostas Anos de inquérito 2008 2013 2019

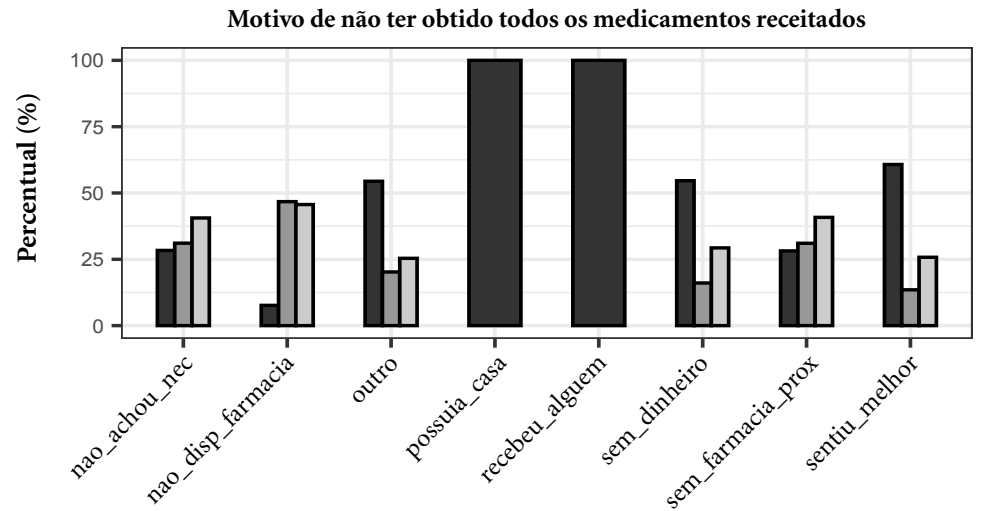

Categorias de respostas Anos de inquérito - 2008 2013 2019

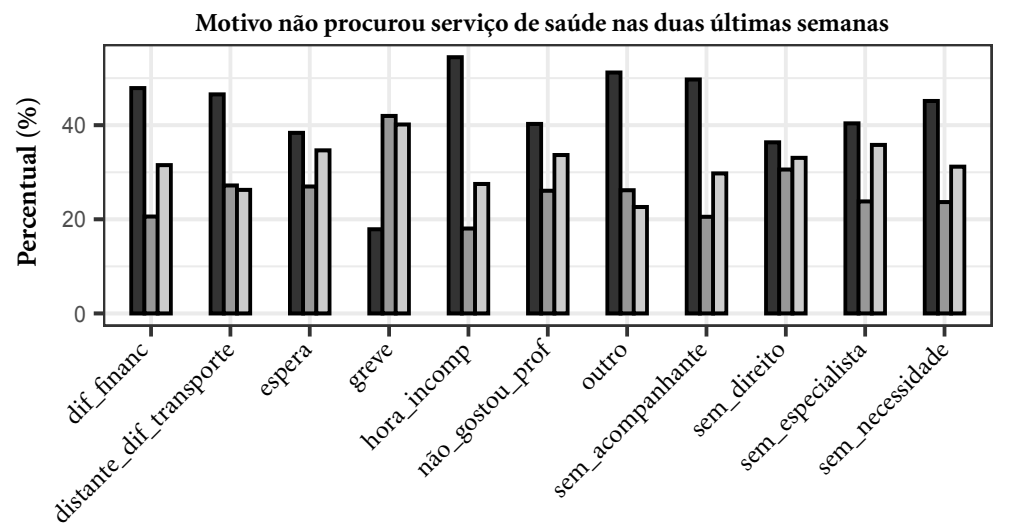

Categorias de respostas Anos de inquérito - 2008 2013 2019

Figura 3. Prevalência de alguns dos motivos de não acesso e utilização de serviços de saúde e não obtenção de medicamentos prescritos, segundo ano do inquérito.

Fonte: Instituto Brasileiro de Geografia e Estatística, Pesquisa Nacional por Amostra de Domicílios 2008, Pesquisa Nacional de Saúde 2013, Pesquisa Nacional de Saúde 2019. 
os serviços de saúde e economia das famílias. Os inquéritos de base populacional são de grande importância na análise do perfil de morbidade, prevalência de exposição aos fatores de risco e proteção das DCNT, assim como no conhecimento do acesso à assistência à saúde ${ }^{8}$. Com base em três inquéritos populacionais realizados entre 2008 e 2019, este estudo avaliou as mudanças das prevalências das DCNT, condições de saúde, e acesso e utilização dos serviços, considerando a influência de variáveis sociodemográficas.

A comparação da PNAD 2008 e PNS 2013 e 2019 deve ser realizada com cautela, pois esses inquéritos utilizaram metodologias diferentes $^{15-17}$, no entanto, utilizando adequadamente desenhos amostrais e metodologia de análise, é possível extrair informações relevantes para a Saúde Pública. Na comparação das morbidades crônicas entre os três inquéritos utilizados, houve aumento de todas as DCNT, com exceção de câncer de intestino e estômago, angina, insuficiência cardíaca, alguma doença pulmonar, IRC e DORT.

A elevação da frequência destas DCNT pode relacionar-se ao envelhecimento da população brasileira associada às mudanças nos hábitos $\mathrm{e}$ estilo de vida, ocorridos com o processo de urbanização e industrialização do país. Anualmente em todo o mundo, ocorrem cerca de 6 milhões de mortes devido ao tabaco, associado a $70,00 \%$ dos cânceres de pulmão, $42,00 \%$ das DRC, e 10,00\% das DAC. A inatividade física é atribuída a 3,2 milhões de mortes, e ao aumento de $20,00 \%$ a $30,00 \%$ de todas as causas de morte. O uso nocivo de álcool é responsável por aproximadamente 2,3 milhões de óbitos, sendo 50,00\% associados às $\mathrm{DCNT}^{6,7}$. A ampliação do acesso aos exames diagnósticos clínicos, laboratoriais e ao tratamento possibilitou a identificação destas morbidades na população, assim como o aumento da sobrevida dos indivíduos acometidos por essas doenças, especialmente, DM, IAM, AVC e câncer ${ }^{9,11-12}$.

Ter recebido diagnóstico de uma condição clínica por profissional de saúde ou seguir tratamento medicamentoso para o controle de uma doença, implicam em ter tido acesso aos serviços de saúde. O aumento da prevalência de uma doença pode ser um indicador positivo de acesso a serviços, como a ampliação do acesso a exames e testes de diagnóstico ${ }^{25}$.

No Brasil, após a implantação do SUS, observa-se melhoria nas condições de saúde da população devido ao aumento do acesso à assistência à saúde, com aumento da proporção de consultas realizada nos últimos 12 meses, cobertura da estratégia de saúde da família, exames complemen- tares, preventivo ginecológico, 95,25\% dos brasileiros que procuram serviço nas últimas duas semanas conseguiram atendimento na primeira vez que buscaram por atendimento ${ }^{18,26}$. Achados que foram corroborados ao avaliar os indivíduos que apresentaram ao menos uma DCNT nos inquéritos populacionais realizados entre 2008 e 2019, pois em relação ao ano de 2008 quando ajustou-se os modelos por região de residência, sexo e faixa etária, verificou-se aumento da prevalência da cobertura da estratégia de saúde família, consulta médica no último ano e procura de atendimento nas duas últimas semanas. A ampliação do acesso aos serviços de saúde pode ter contribuído para o aumento da prevalência de brasileiros que autorreferiram sua saúde como boa ou muito boa, redução da prevalência da limitação de atividades de rotina e de brasileiros que estiveram acamados no último ano, assim como redução da prevalência de internação nos últimos 12 meses.

Em contrapartida, verificou-se menor prevalência de atendimento na primeira procura em indivíduos que buscaram atendimento nas duas últimas semanas e aumento da prevalência de atendimentos realizados por plano de saúde (2019 versus 2008), somado a menor prevalência de obtenção de medicamentos em serviço público e no programa de farmácia popular. Situação que pode estar associada ao menor orçamento direcionado para a Saúde após o Projeto de Emenda Constitucional 95 que reduziu o orçamento para Seguridade Social, e com o isso os recursos para o SUS. O Brasil optou por diminuir o financiamento da saúde em um momento de acelerado envelhecimento populacional e aumento das DCNT que exigem maior desempenho do sistema de saúde, por serem morbidades com tratamentos dispendiosos e de longa duração, e com alta probabilidade de gerar limitações ${ }^{8}$.

Os inquéritos populacionais de desenho transversal permitem o estudo dos casos (doenças/condições de saúde) prevalentes, e assim características correlacionadas com a sobrevivência serão mais frequentes entre os casos (viés de sobrevivência). Portanto, as maiores prevalências de DAC, cânceres, DM, IRC e doenças respiratórias crônicas em área urbana, e nas regiões mais desenvolvidas do país, podem ser explicadas tanto pelo viés de sobrevivência quanto pela estrutura etária nessas localidades. As regiões mais desenvolvidas apresentam menor iniquidade no acesso à assistência à saúde, reduzindo a letalidade dessas morbidades ${ }^{18,26}$, e apresentam estrutura etária mais envelhecida. 
As prevalências de DAC, CA, IRC, artrite reumatoide, DM, alguma doença do pulmão, asma e/ou bronquite apresentaram diferenças segundo raça/cor e escolaridade, com maior prevalência em indivíduos brancos e com baixo nível de escolaridade. No Brasil, existem iniquidades em saúde e essas estão associadas à classe e a raça, negros e pobres acessam menos os serviços de saúde, devido barreiras estruturais, socioeconômicas, dificuldade dos profissionais em lidar com a diversidade racial e de classe ${ }^{27,28}$. Os indivíduos com baixa escolaridade possivelmente podem apresentar maior prevalência dessas morbidades com exceção da asma e/ou bronquite, pois estão menos expostos aos fatores de proteção como alimentação saudável, prática de atividade física e acesso aos serviços de saúde. A utilização dos serviços de saúde foi mais prevalente em pessoas com maior escolaridade, inversamente o relato de discriminação nos serviços de saúde foi mais frequente em pessoas de baixa escolaridade ${ }^{28,29}$.

A redução observada na prevalência de IRC pode estar associada ao maior controle da HAS e DM devido à ampliação do acesso à atenção básica e ao acesso aos medicamentos ${ }^{25,29}$. Apesar da redução observada na frequência dessa condição de saúde entre os inquéritos, a presença dessa condição de saúde é considerada alta (três a seis milhões de brasileiros), por cursar com alto grau de incapacidade e por seu tratamento ter alto custo para o SUS ${ }^{8,30}$. Estima-se um aumento de $66,66 \%$ do número de brasileiros em tratamento de hemodiálise no período 2005 a 2015, e 84,4\% dos tratamentos são custeados pelo SUS ${ }^{30}$.

A artrite reumatoide e dor crônica na coluna e nas costas foram as condições crônicas mais relatadas por brasileiros na PNAD 2003 e 2008 superadas apenas pela $\mathrm{HAS}^{25}$, ainda dores na coluna lombar e cervical foram a principal causa de anos vividos com incapacidade no Brasil nos anos de 1990 e $2016^{1}$. A perda da funcionalidade aumenta os custos em saúde devido à necessidade de consultas médicas com especialistas, gastos com medicações analgésicas muitas vezes de alto custo, atendimento fisioterápico, além do impacto na previdência social devido ao afastamento remunerado e aposentadoria precoce ${ }^{29-31}$. Em 2013, essas morbidades e a DORT foram responsáveis por $49,20 \%$ das doenças que promovem limitações muito intensas/ intensas para atividades de vida diária ${ }^{8}$, associando-se ao sedentarismo e depressão, e desta maneira aumentando o risco de desenvolvimento de outras $\mathrm{DCNT}^{8,29,32}$. Os principais fatores associados a essas condições foram ser do sexo feminino, ter sobrepeso ou obesidade, desempenhar atividade doméstica e/ou no trabalho com alta carga muscular ${ }^{29,32}$. No entanto, há diferenças na prevalência de Dor na coluna (DC) e nas costas e DORT em relação às variáveis nível de escolaridade e zona de residência. Indivíduos com menor nível de escolaridade e residentes em zona rural apresentam maior prevalência de DC, devido aos trabalhos mais exaustivos que desempenham ${ }^{32}$. A maior prevalência de DORT nas regiões mais desenvolvidas do país e em área urbana, pode associar-se às especificidades do processo de trabalho nessas localidades, e a maior possibilidade de ter acesso à medicina do trabalho para o diagnóstico de morbidades associadas ao trabalho ${ }^{32}$.

Outra condição crônica que se destaca no perfil epidemiológico da população brasileira entre os três inquéritos são as doenças neuropsiquiátricas, com destaque para a depressão. Discute-se que o aumento da carga das DN na população brasileira esteja relacionado às mudanças sociodemográficas e econômicas que ocorreram em nosso país nas últimas décadas, marcadas por crises econômicas e fragilização das relações de trabalho. Neste contexto, destacase a urbanização acelerada e não planejada, em que grandes contingentes populacionais residem em territórios de grande vulnerabilidade social, forte presença do crime organizado e exposição à violência e ausência do Estado ${ }^{33-35}$.

\section{Conclusão}

As DN e o uso de substâncias psicoativas apresentam grandes contribuições para carga de doenças no Brasil, em 2015 contribuíram para 9,30\% dos anos de vida perdidos por incapacidade (DALY) ${ }^{36}$. Refletindo a importância das DN na carga de doença brasileira, passando da sexta posição em 1990 para a terceira posição em 2015, sendo superada somente pela DAC e cânceres ${ }^{33}$. Corroborando essas evidências, no presente estudo observou-se aumento das DN entre os inquéritos analisados, com maior prevalência no ano de 2019 (11,61\%) com destaque para depressão que contribuiu com $88,20 \%$ das DN nesse período.

A interação entre transtornos mentais e outras doenças crônicas é discutido na literatura, alguns transtornos podem surgir como consequência das incapacidades geradas pelas DCNT ou apresentarem-se como fatores de risco para esse conjunto de doenças ${ }^{33,34}$, pois indivíduos acometidos por DN apresentam maior risco de adota- 
rem hábitos e estilos de vida não saudáveis tais como alimentação inadequada, sedentarismo, consumo excessivo de álcool ${ }^{35}$, o que pode explicar o aumento do risco de eventos coronários em indivíduos que apresentam depressão $0^{33,34}$. Sinalizando a importância do tratamento dos transtornos mentais, no entanto o SUS apresenta barreiras para o acompanhamento dessas morbidades, ainda há forte estigma social que dificulta a busca e a adesão ao tratamento, somado a quantidade inadequada de Centro de Atenção Psicossocial (CAPS) e a falta de treinamento da equipe da atenção básica para o acolhimento e tratamento desses usuários ${ }^{33}$.

\section{Colaboradores}

Todos os quatro autores TC Simões, KC Meira, J Santos, DCP Câmara contribuíram em todos os pontos: concepção e o delineamento ou a análise e interpretação dos dados; redação do artigo ou a sua revisão crítica; e aprovação da versão a ser publicada.
Dentre as limitações do estudo citam-se as diferenças das unidades amostrais entre PNAD e PNS, assim como mudanças nas variáveis coletadas, perguntas dos questionários e categorias de resposta que demandam um grande esforço inicial de harmonização e compatibilização das bases (metodológico e de processamento de software), que não são detalhadamente descritos em outros estudos, dificultando a comparabilidade das estimativas. Há ainda, o conhecido problema de viés de sobrevivência e a causalidade reversa inerentes a estudos transversais. Além disso, a prevalência de morbidades autorrelatadas pode ser influenciada pelo acesso aos serviços de saúde.

\section{Referências}

1. GBD 2019 Risk Factors Collaborators. Global burden of 87 risk factors in 204 countries and territories, 19902019: a systematic analysis for the Global Burden of Disease Study 2019. Lancet 2020; 396(10258):1.223-1.249.

2. Pullar J, Allen L, Townsend N, Williams J, Foster C, Roberts N, Rayner M, Mikkelsen B, Branca F, Wickramasinghe $\mathrm{K}$. The impact of poverty reduction and development interventions on non-communicable diseases and their behavioural risk factors in low and lower-middle income countries: A systematic review. PLoS One 2018; 13(2):e0193378.

3. Oliveira GMM, Brant LCC, Polanczyk AC, Biolo ANBR, Malta DC, Souza MFM, Soares GP, Junior GFX, Machline-Carrion MJ, Bittencourt MS, Neto OMP, Silvestre OM, Teixeira RA, Sampaio RO, Gaziano TA, Roth GA, Ribeiro ALP. Estatística Cardiovascular - Brasil 2020. Arq Bras Cardiol 2020; 115(3):308-439.

4. Nascimento BR, Brant LCC, Oliveira GMM, Malachias MVB, Reis GMA, Teixeira RA, Malta DC, França E, Souza MFM, Roth GA, Ribeiro ALP. Cardiovascular disease epidemiology in portuguese-speaking countries: data from the Global Burden of Disease, 1990 to 2016. Arq Bras Cardiol 2018; 110(6):500-511.

5. Pirani N, Khiavi FF. Population Attributable Fraction for Cardiovascular Diseases Risk Factors in Selected Countries: A comparative study. Mater Sociomed 2017; 29(1):35-39.

6. World Health Organization (WHO). Global health risks: mortality and burden of disease attributable to selected major risks. Geneva: WHO; 2009.

7. World Health Organization (WHO). Noncommunicab le diseases progress monitor 2020. Geneva: WHO; 2020.

8. Malta DC, Stopa SR, Szwarcwald CL, Gomes NL, Silva JJB, Reis AAC. A vigilância e o monitoramento das principais doenças crônicas não transmissíveis no Brasil - Pesquisa Nacional de Saúde, 2013. Rev. bras Epidemiol 2015; 18(Supl. 2):3-16. 
9. Brasil. Ministério da Saúde (MS). Secretaria de Vigilância em Saúde. Saúde Brasil 2018: Principais causas específicas de morte no Brasil e regiões, e mortalidade prematura por DCNT, 2005 a 2016. Uma análise da situação de saúde e das doenças e agravos crônicos: desafios e perspectivas. Brasília: MS; 2019.

10. Khan MA, Hashim MJ, Mustafa H, Baniyas MY, Al Suwaidi SKBM, AlKatheeri R, Alblooshi FMK, Almatrooshi MEAH, Alzaabi MEH, Al Darmaki RS, Lootah SNAH. Global Epidemiology of Ischemic Heart Disease: Results from the Global Burden of Disease Study. Cureus 2020;12(7):e9349.

11. Viacava F, Oliveira RAD, Carvalho CC, Laguardia J, Bellido JG. SUS: oferta, acesso e utilização de serviços de saúde nos últimos 30 anos. Cien Saude Colet 2018; 23(6):1.751-1.762.

12. Malta DC, Teixeira R, Oliveira GMM, Ribeiro ALP. Mortalidade por doenças cardiovasculares segundo o Sistema de Informação sobre Mortalidade e as estimativas do estudo Carga Global de Doenças no Brasil, 2000-2017. Arq Bras Cardiol 2020; 115(2):152-160.

13. Brasil. Ministério da Saúde (MS). Plano de ações estratégicas para o enfrentamento das doenças crônicas não transmissiveis (DCNT) no Brasil, 2011-2022. Brasília: MS; 2011.

14. Malta DC, Leal MC, Costa MFL, Neto OLM. Inquéritos Nacionais de Saúde: experiência acumulada e proposta para o inquérito de saúde brasileiro. Rev Bras Epidemiol 2008; 11(Supl. 1): 159-67.

15. Instituto Brasileiro de Geografia e Estatística (IBGE). Pesquisa Nacional por Amostra de Domicílios. Panorama da Saúde no Brasil: acesso e utilização dos serviços, condições de saúde e fatores de risco e proteção à saúde (PNAD 2008). Rio de Janeiro: IBGE; 2010.

16. Instituto Brasileiro de Geografia e Estatística (IBGE). Pesquisa Nacional de Saúde 2013: Acesso e utilização dos serviços de saúde, acidentes e violências: Brasil, Grandes Regiões e Unidades da Federação. Rio de Janeiro: IBGE; 2015.

17. Stopa SR, Szwarcwald CL, Oliveira MM, Gouvea ECDP, Vieira MLFP, Freitas MPS, Sardinha LMV, Macário EM. Pesquisa Nacional de Saúde 2019: histórico, métodos e perspectivas. Epidemiol Serv Saude 2020; 29(5):e2020315.

18. Viacava F, Bellido JG. Condições de saúde, acesso a serviços e fontes de pagamento, segundo inquéritos domiciliares. Cien Saude Colet 2016; 21(2):351-370.

19. Lee S, Davis W, Anh Nguyen H, McNeel T, Brick J, Flores Cervantes I. Examining Trends and Averages Using Combined Cross Sectional Survey Data from Multiple Years. Los Angeles, CA: UCLA Center for Health Policy Research; 2006.

20. Rao JNK, Scott AJ. On Chi-Squared Tests for Multiway Contingency Tables with Cell Proportions Estimated from Survey Data. The Annals of Statistics 1984; 12(1):46-60.

21. Barros AJ, Hirakata VN. Alternatives for logistic regression in cross-sectional studies: an empirical comparison of models that directly estimate the prevalence ratio. BMC Med Res Methodol 2003; 3(1):21.

22. Silva PLN, Pessoa DGC, Lila MF. Análise estatística de dados da PNAD: incorporando a estrutura do plano amostral. Cein Saude Colet 2002; 7(4):659-670.

23. Lumley T. Complex Surveys: A Guide to Analysis Using R. Hoboken, N.J.: John Wiley; 2010.
24. Foundation for Statistical Computing. $R$ Core Team. $R$ : A language and environment for statistical computing. Vienna; 2020.

25. Barros MBA, Francisco PMSB, Zanchetta LM, César CLG. Tendências das desigualdades sociais e demográficas na prevalência de doenças crônicas no Brasil, PNAD: 2003- 2008. Cien Saude Colet 2011; 16(9):3.755-3.768.

26. Macinko J, Mendonça. Estratégia Saúde da Família, um forte modelo de Atenção Primária à Saúde que traz resultados. Saude Debate 2018; 42(n. esp.):18-37.

27. Silva NN, Favacho VBC, Boska GA, Andrade EC, Merces NP, Oliveira MAF. Acesso da população negra a serviços de saúde: revisão integrativa. Rev Bras Enferm 2020; 73(4):e20180834.

28. Boccolini CS, Boccolini PMM, Damacena GN, Ferreira APS, Szwarcwald CL. Fatores associados à discriminação percebida nos serviços de saúde do Brasil: resultados da Pesquisa Nacional de Saúde, 2013. Cein Saude Colet 2016;21(2):371-378.

29. Malta DC, Bernal RTI, Lima MG, Araújo SSC, Silva MMA, Freitas MIF, Barros MBA. Doenças crônicas não transmissíveis e a utilização de serviços de saúde: análise da Pesquisa Nacional de Saúde no Brasil. Rev Saude Publica 2017; 51(Supl. 1):4s.

30. Marinho AWGB, Penha AP, Silva MT, Galvão TF. Prevalência de doença renal crônica em adultos no Brasil: revisão sistemática da literatura. Cad Saude Colet 2017; 25(3):379-388.

31. Assunção AA, Abreu MNS. Fatores associados a distúrbios osteomusculares relacionados ao trabalho autorreferidos em adultos brasileiros. Rev Saude Publica 2017; 51(Supl. 1):10s.

32. Malta DC, Oliveira MM, Andrade SSCA, Caiaffa WT, Souza MFM, Bernal RTI. Fatores associados à dor crônica na coluna em adultos no Brasil. Rev Saude Publica 2017; 51(Supl. 1):9s.

33. Lopes CS. Como está a saúde mental dos brasileiros? A importância das coortes de nascimento para melhor compreensão do problema. Cad Saude Publica 2020; 36(2): e00005020.

34. Bonadiman CSC, Passos VMA, Mooney M, Naghavi M, Melo APS. A carga dos transtornos mentais e decorrentes do uso de substâncias psicoativas no Brasil: Estudo de Carga Global de Doença, 1990 e 2015. Rev Bras Epidemiol 2017; 20(Supl. 1): 191-204.

35. Barros MBA, Lima MG, Azevedo RCS, Medina LBP, Lopes CS, Menezes PR, Malta DC. Depressão e comportamentos de saúde em adultos brasileiros - PNS 2013. Rev Saude Publica 2017; 51(Supl. 1):8s.

36. Costa PHA, Colugnati FAB, Ronzani TM. Avaliação de serviços em saúde mental no Brasil: revisão sistemática da literatura. Cien Saude Colet 2015; 20(10):3.243-3.245.

Artigo apresentado em 02/02/2021

Aprovado em 22/03/2021

Versão final apresentada em 24/03/2021

Editores chefes: Maria Cecília de Souza Minayo, Romeu Gomes, Antônio Augusto Moura da Silva 